\title{
Two Cases of Tsunami Dust Pneumonia: Organizing Pneumonia Caused by the Inhalation of Dried Tsunami Sludge after the 2011 Great East Japan Earthquake
}

\author{
Shinsuke Yamanda ${ }^{1}$, Seiichi Kobayashi ${ }^{1}$, Masakazu Hanagama ${ }^{1}$, Hikari Sato ${ }^{1}$, \\ Satoshi Suzuki ${ }^{2}$, Shinsaku Ueda ${ }^{2}$, Toru Takahashi ${ }^{3}$ and Masaru Yanai ${ }^{1}$
}

\begin{abstract}
We report two cases of organizing pneumonia (OP) secondary to the inhalation of the dried tsunami sludge which formed during the 2011 Great East Japan Earthquake and the consequent tsunami. After the disaster, both of these patients had been engaged in the restoration work. About half a month later, they developed shortness of breath and pulmonary infiltrates. These patients were diagnosed with interstitial pneumonia. Their biopsy specimens revealed multifocal peribronchiolitis and OP. An electron probe microanalysis of these specimens demonstrated the presence of elements from the earth's crust in the inflammatory lesions. These two cases indicate that exposure to dried tsunami sludge can cause OP.
\end{abstract}

Key words: earthquake, tsunami, organizing pneumonia, electron probe microanalysis (EPMA)

(Intern Med 55: 3645-3653, 2016)

(DOI: 10.2169/internalmedicine.55.6952)

\section{Background}

Organizing pneumonia (OP) is defined histopathologically by the presence of intraluminal polyps of connective tissue in the distal air spaces and it is distinct from the histopathology of minor interstitial fibrosis (1). This histological pattern is not specific to any disorder, but it reflects a type of inflammatory process caused by lung injury. Its idiopathic form is called "cryptogenic organizing pneumonia (COP)", and the cases with some particular cause or associated disease are called "secondary organizing pneumonia."

Secondary OP has various causes, such as infection, aspiration drug reaction, diffuse alveolar damage, collagen vascular disease, and organ transplantation (1). Dust inhalation is also known to cause OP. However, the development of OP by the inhalation of dried tsunami sludge has not yet been reported.

Our patients were engaged in the restoration work after the 2011 Great East Japan Earthquake that struck the shores of the Gulf of Ishinomaki. Therefore, the lung disease in these patients may be attributable to the inhalation of an enormous amount of dust containing dried tsunami sludge during the restoration work. A few studies have reported the development of interstitial pneumonia after a major disaster or accident; however, no studies have reported any cases during restoration activities. Moreover, the reactive agent and its mechanism of action remain unclear. We herein report two cases of OP, show the histopathology of lung specimens and the results of their elemental analysis, and also discuss the role of air pollution in this disease process after the tsunami.

\section{Case Reports}

\section{Case 1}

In May of 2011, a 58-year-old man living in Ishinomaki, located on the northeast coast of the main island of Japan facing the Pacific Ocean, was admitted to our hospital, with a complaint of progressive dyspnea and a productive cough for the past 10 days. His house had been severely damaged

${ }^{1}$ Department of Respiratory Medicine, Japanese Red Cross Ishinomaki Hospital, Japan, ${ }^{2}$ Department of Thoracic Surgery, Japanese Red Cross Ishinomaki Hospital, Japan and ${ }^{3}$ Department of Pathology, Japanese Red Cross Ishinomaki Hospital, Japan

Received for publication December 9, 2015; Accepted for publication May 1, 2016

Correspondence to Dr. Shinsuke Yamanda, syamanda@gmail.com 

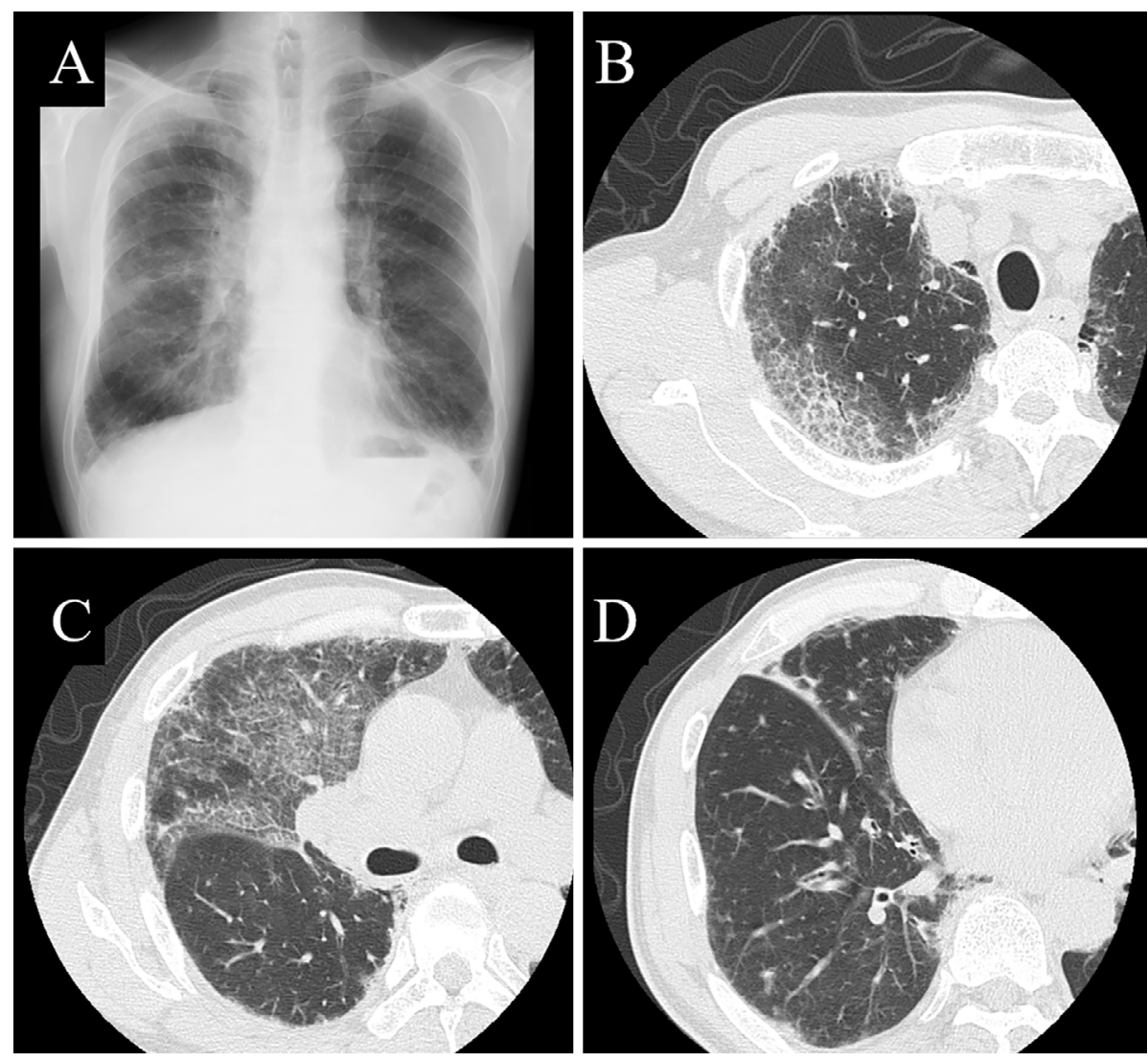

Figure 1. Chest radiograph (A) and high-resolution chest CT scan (lung windowing) of the right lobe of the lung (B-D) in case 1.

and deeply covered with seabed sludge after the devastating earthquake and the tsunami that struck northeastern Japan on March 11, 2011. He had been engaged in the removal of sludge and also the cleaning and repair of his house without using any airway protective device, such as a dust protective mask, for two weeks before hospital admission. His medical history was remarkable for autoimmune hepatitis, which was controlled with a low dose of prednisolone (5 mg per day) and he had been free from any bronchial asthma attacks for 12 years. He had a smoking history of 20 pack-years and had quit smoking immediately after presenting with these symptoms. He did not have occupational exposure to dust.

Upon clinical examination, he had a temperature of $38.2^{\circ} \mathrm{C}$ and pulse oximetry saturation of $90 \%$ in room air. $\mathrm{He}$ presented with bilateral end-inspiratory fine crackles without wheezing. Blood tests indicated an increase in Creactive protein $(14.9 \mathrm{mg} / \mathrm{dL})$ and a white blood cell count $\left(11.0 \times 10^{9} / \mathrm{L} ; 79 \%\right.$ neutrophils, $0.7 \%$ eosinophils). The levels of liver enzymes increased slightly (aspartate aminotransferase of $82 \mathrm{IU} / \mathrm{L}$, alanine aminotransferase of $86 \mathrm{IU} / \mathrm{L}$ ), but they were similar to normal values. His levels of serum KL6 , which is a marker of pulmonary fibrosis, were remarkably elevated $(1,630 \mathrm{U} / \mathrm{mL})$. Chest radiography revealed the presence of bilateral reticular opacities (Fig. 1A), and a chest computed tomography (CT) scan demonstrated the predominance of ground-glass opacities and interlobular septal thickening in the periphery of the upper lobes (Fig. 1B-D). The respiratory function test demonstrated a mild restrictive disorder, vital capacity (VC) of $2.85 \mathrm{~L}(79.8 \%)$, and a forced expiratory volume in one second/forced vital capacity (FEV1/FVC) of $70.6 \%$.

The patient was diagnosed with community-acquired pneumonia and was treated with ceftriaxone and azithromycin. However, his symptoms did not improve, and the area and density of lung opacities increased. He underwent video-assisted thoracoscopic surgical (VATS) lung biopsy. Histopathology showed multifocal peribronchiolitis. A single focus measured approximately $5 \mathrm{~mm}$ in diameter, roughly corresponding to an acinus, and contained air spaces filled with macrophages, plasma cells, lymphocytes, and neutrophils. In the periphery of this focus, alveolar ducts were obstructed by nodular fibrous plugs, which are known as Masson bodies (Fig. 2). The airways at the center of this focus contained primarily terminal or respiratory bronchioles and were patent and aerated. These findings indicated the presence of OP had been caused by the inhalation of noxious particles.

\section{Case 2}

In May of 2011, a 63-year-old man living in HigashiMatsushima, which is adjacent to Ishinomaki and face the Pacific Ocean, was admitted to our hospital with a progression of dyspnea on exertion. His house had been damaged by the tsunami and was covered with seabed sludge. He had 


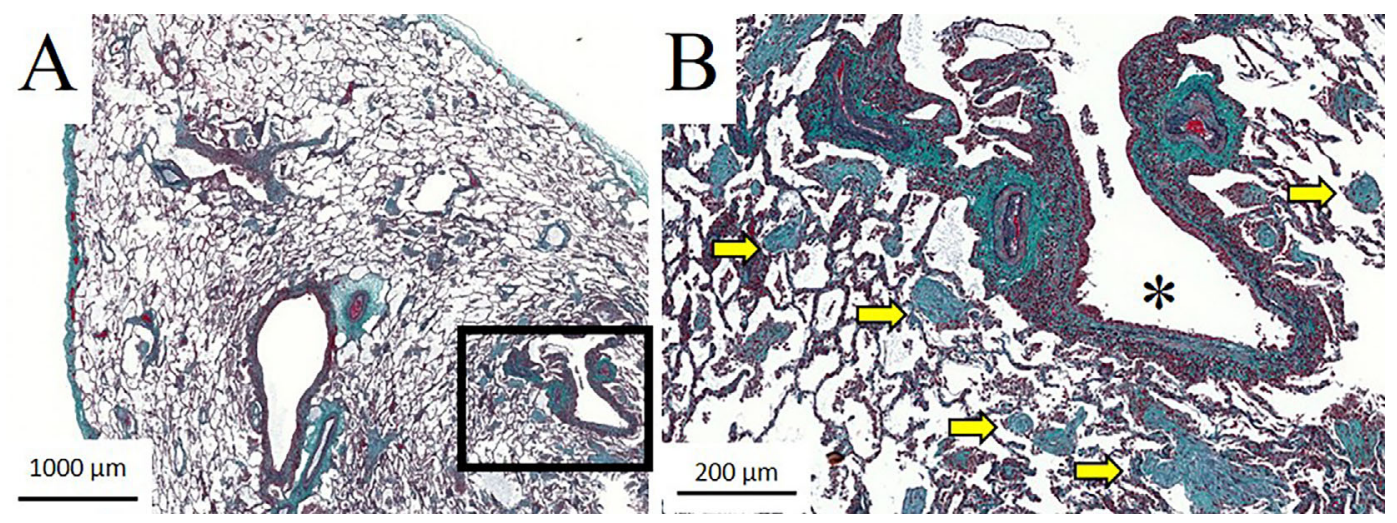

Figure 2. An open lung biopsy specimen obtained from the periphery of the anterior segment of the right upper lobe (right S3) and stained with Elastica-Masson in the low-power field (A) and highpower field $(B)$ in case 1 . The intraluminal accumulation of macrophages and the focal proliferation of nodular fibrous plugs (arrow) are observed around the respiratory bronchioles (asterisk).
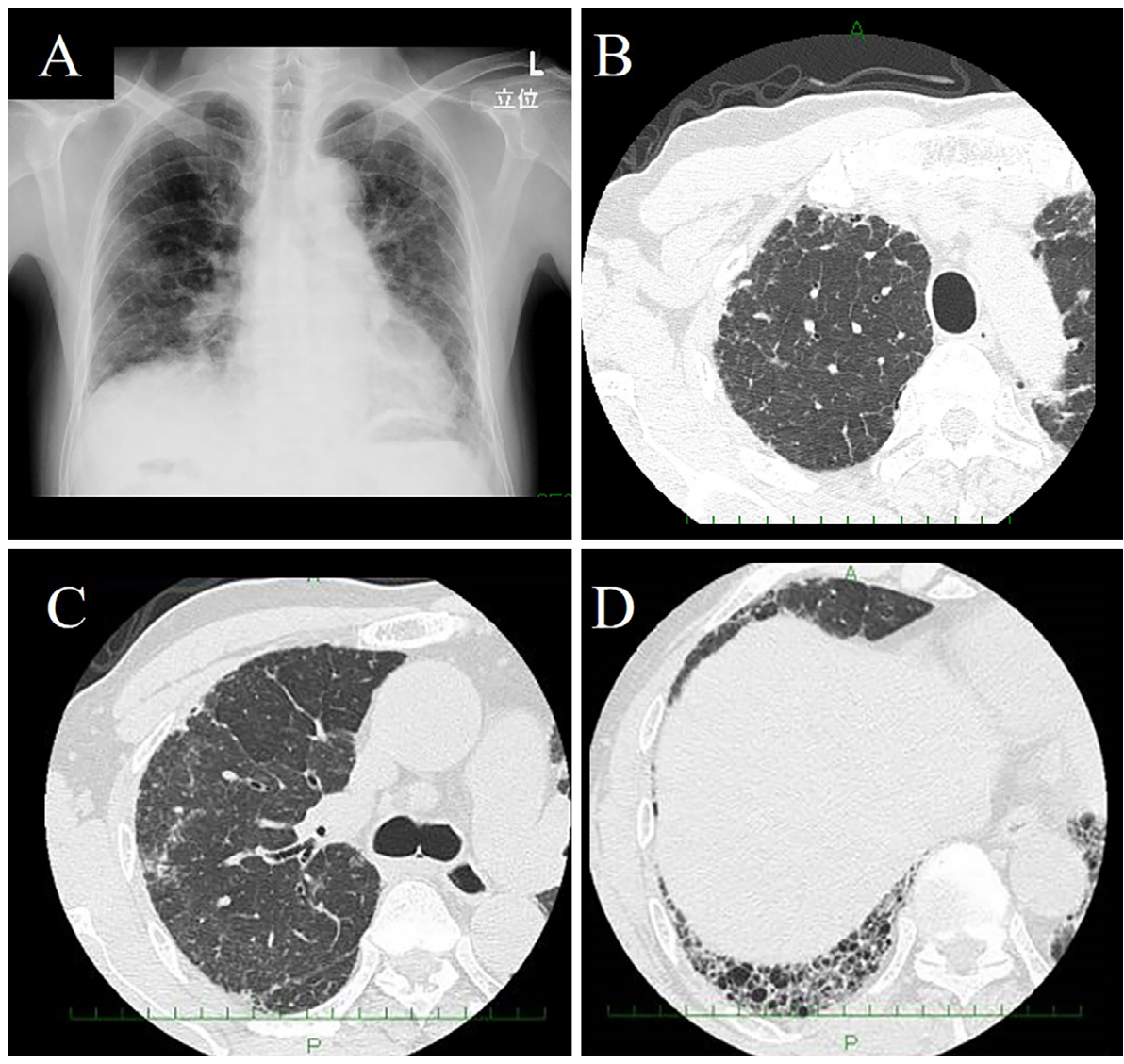

Figure 3. Chest radiograph (A) and high-resolution chest CT scan (lung windowing) of the right lobe of the lung (B-D) in case 2.

been engaged in the removal of sludge and in cleaning the house without any airway protective device before hospital admission. His medical history was remarkable for squamous cell lung carcinoma, which had been treated with photodynamic therapy for 10 years, and for idiopathic pulmonary fibrosis (IPF), which had been clinically diagnosed three years before this hospital admission and had been treated with an antitussive agent. He had a smoking history of 30 pack-years and had quit smoking 10 years before this hospital admission. He had worked as a construction worker for 40 years.

Upon clinical examination, the patient was afebrile, and his pulse oximetry saturation was $92 \%$ in room air. He presented with bilateral end-inspiratory fine crackles and clubbed fingers; however, these findings had all been recorded during a previous visit to the hospital. Blood tests indicated a slight increase in the C-reactive protein level (1.8 $\mathrm{mg} / \mathrm{dL}$ ) without any elevation of the white blood cell count, 


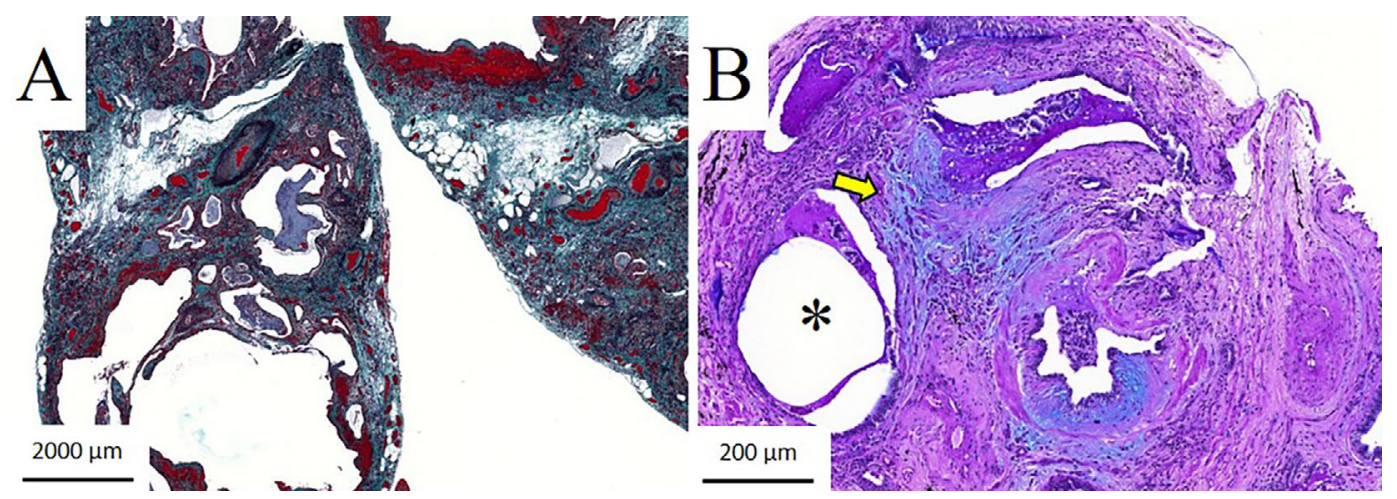

Figure 4. An open lung biopsy specimen obtained from the posterior basal segment of the right lower lobe (right S10) and stained with Elastica-Masson (A) and Alcian blue periodic acid Schiff (B) in case 2. Interstitial thickening, honeycombing (asterisk), and fibroblastic foci (arrow) can be observed.
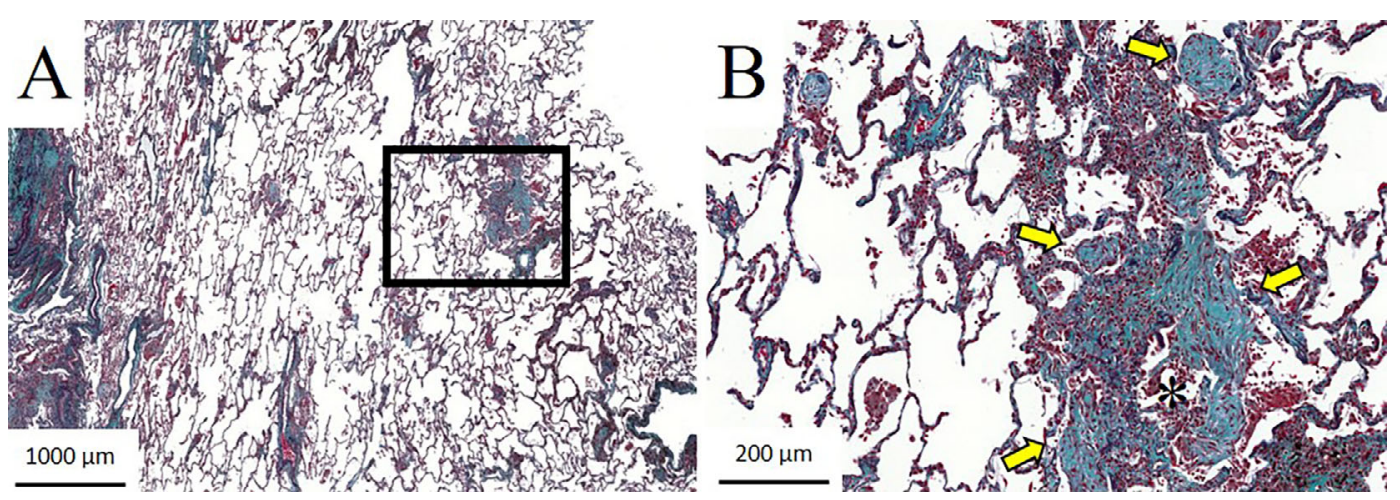

Figure 5. An open lung biopsy specimen obtained from the anterior segment of the right upper lobe (right S3) and stained with Elastica-Masson in the low-power field (A) and high-power field (B) in case 2. The intraluminal accumulation of macrophages and the focal proliferation of nodular fibrous plugs (arrow) are observed around the respiratory bronchioles (asterisk).

while the liver and renal functions were normal. His serum levels of KL-6 $(1,230 \mathrm{U} / \mathrm{mL})$ had increased above the usual levels. Chest radiography revealed the presence of interstitial shadows in both lung fields and an elevation of the diaphragm (Fig. 3A). A chest CT scan demonstrated the presence of fine bilateral nodular shadows in the middle lobe, and peripheral honeycomb cysts with lung consolidation in the lower lobe (Fig. 3B-D). Although the diagnosis of the latter complication had been confirmed in a previous chest CT scan, the respiratory function test demonstrated severe restrictive disorder, $\mathrm{VC}$ of $1.31 \mathrm{~L}$ (37.9\%), and FEV1/FVC of $87.0 \%$.

The patient was suspected of having developed acute exacerbation of IPF and, therefore, a VATS lung biopsy was performed. Microscopically, a usual interstitial pneumonia (UIP) pattern was observed in the lower and upper lobes of the right lung, and this pattern was characterized by interstitial fibrosis, fibroblastic foci, and moderately advanced honeycombing (Fig. 4). In addition, non-remodeling areas presented changes in the OP pattern, with the accumulation of various inflammatory cells around the bronchioles, obstruction of the peripheral alveolar ducts with Masson body, and the presence of terminal or respiratory bronchioles, which were all findings similar to those observed in case 1 (Fig. 5). The patient was not diagnosed with acute exacerbation of IPF, but instead was diagnosed with OP due to the inhalation of noxious particles and with IPF. We treated the patient with a daily dose of $60 \mathrm{mg}$ of prednisolone.

Their sputum cultures were free of pathogens and, for this reason, we suspected that OP had been caused by the inhalation of dried sludge from the tsunami. Therefore, we performed an elemental analysis of lung tissue specimens using an electron probe microanalyzer (EPMA) and a wavelengthdispersive spectrometer. In case $1, \mathrm{Fe}, \mathrm{Si}, \mathrm{Al}$, and $\mathrm{Ca}$ were primarily detected in the peribronchioles and alveolar walls (Fig. 6) and these elements coexisted with a relatively large amount of oxygen. It has been suggested that these elements occur in the form of oxides. In case $2, \mathrm{Fe}, \mathrm{Si}, \mathrm{Al}$, and $\mathrm{Ca}$ were primarily detected in the peribronchioles and alveolar walls in the OP zone (Fig. 7) and the same elements were detected in the UIP zone. Similar to what was observed in case 1, a high amount of oxygen coexisted with these elements. However, no heavy metals were detected in any of the cases. 

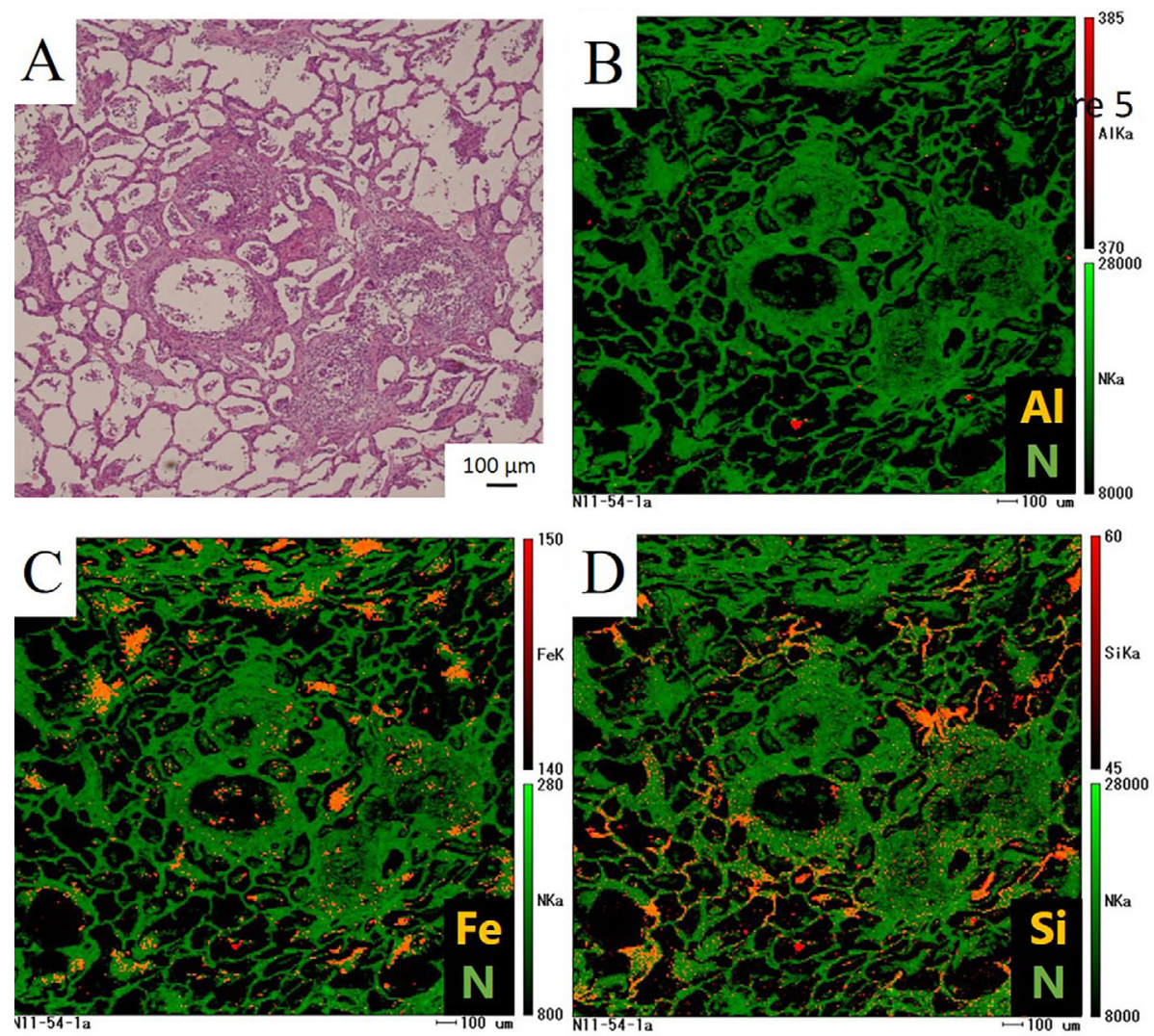

Figure 6. Anelectron probe microanalysis of the periphery of the anterior segment of the right upper lobe in case 1. (A) Hematoxylin and Eosin staining. The deposition of aluminum (B), iron (C), and silicon $(D)$ is shown in yellow.

After histological diagnosis of OP, we treated both patients with prednisolone at $0.5 \mathrm{mg} / \mathrm{kg}$. In case 1 , his symptoms gradually improved (Fig. 8). After two months of treatment, his chest radiograph and respiratory function returned to normal, and the KL-6 levels decreased to $600 \mathrm{U} / \mathrm{mL}$. The symptoms of case 2 gradually improved and the fine nodular shadows identified on the CT scan resolved after two months of treatment (Fig. 9). Neither of the patients had a relapse after the withdrawal of treatment.

\section{Discussion}

On March 11, 2011, a massive earthquake followed by a tsunami hit the northeastern coast of Japan. The city of Ishinomaki experienced the greatest damage, with the inundation of $46 \%$ of its area by the tsunami, and most of the hospitals and clinics in the city were either destroyed or became inoperative. The Japanese Red Cross Ishinomaki Hospital was the only functioning hospital in Ishinomaki. At this hospital, we treated more than 10,000 patients in the first month after the disaster and many others afterward, and also treated a considerable number of pneumonia cases (2). Although most of the patients with pneumonia recovered after receiving antibiotic therapy, the two cases reported herein did not respond to antibiotics, but instead improved after being administered steroid therapy. These two cases have several similarities. Both the patients were engaged in the re- moval of dried sludge from the tsunami and had inhaled this material before onset, and their lung specimens indicated the accumulation of inflammatory cells in the peribronchioles as well as fibrous plugs in the alveolar ducts, which indicated the presence of OP. No bacterial pathogens were detected in the lung samples from either patient. Both of them also showed a favorable response to steroid therapy. Therefore, we hypothesized that these two cases of OP had thus been caused by the inhalation of highly concentrated amounts of dried sludge from the tsunami during the restoration work.

The dried sludge that these two patients inhaled was composed primarily of earth and sand from the seabed, in addition to microorganisms, organic compounds, oils, heavy metals, and chemical compounds such as dioxins and polyvinyl chloride. We did not detect any particular cause of OP and believed that the size and concentration of the dust had most likely strongly influenced the development of OP. An elemental analysis primarily indicated the presence of $\mathrm{Fe}, \mathrm{Si}$, $\mathrm{Al}$, and $\mathrm{Ca}$, with large amounts of oxygen in the peribronchioles and alveolar walls; however, no other elements were found. The size of these earth elements in the atmosphere is usually 5 to 30 micrometers and most of them become trapped in the upper respiratory tract, but only those smaller than 5 micrometers reach the bronchioles and alveoli (3). A study on the constitution of the tsunami sludge indicated the presence of approximately $10 \%$ to $80 \%$ of silt and clay in the elements with a size of less than 7.5 micrometers (4). 

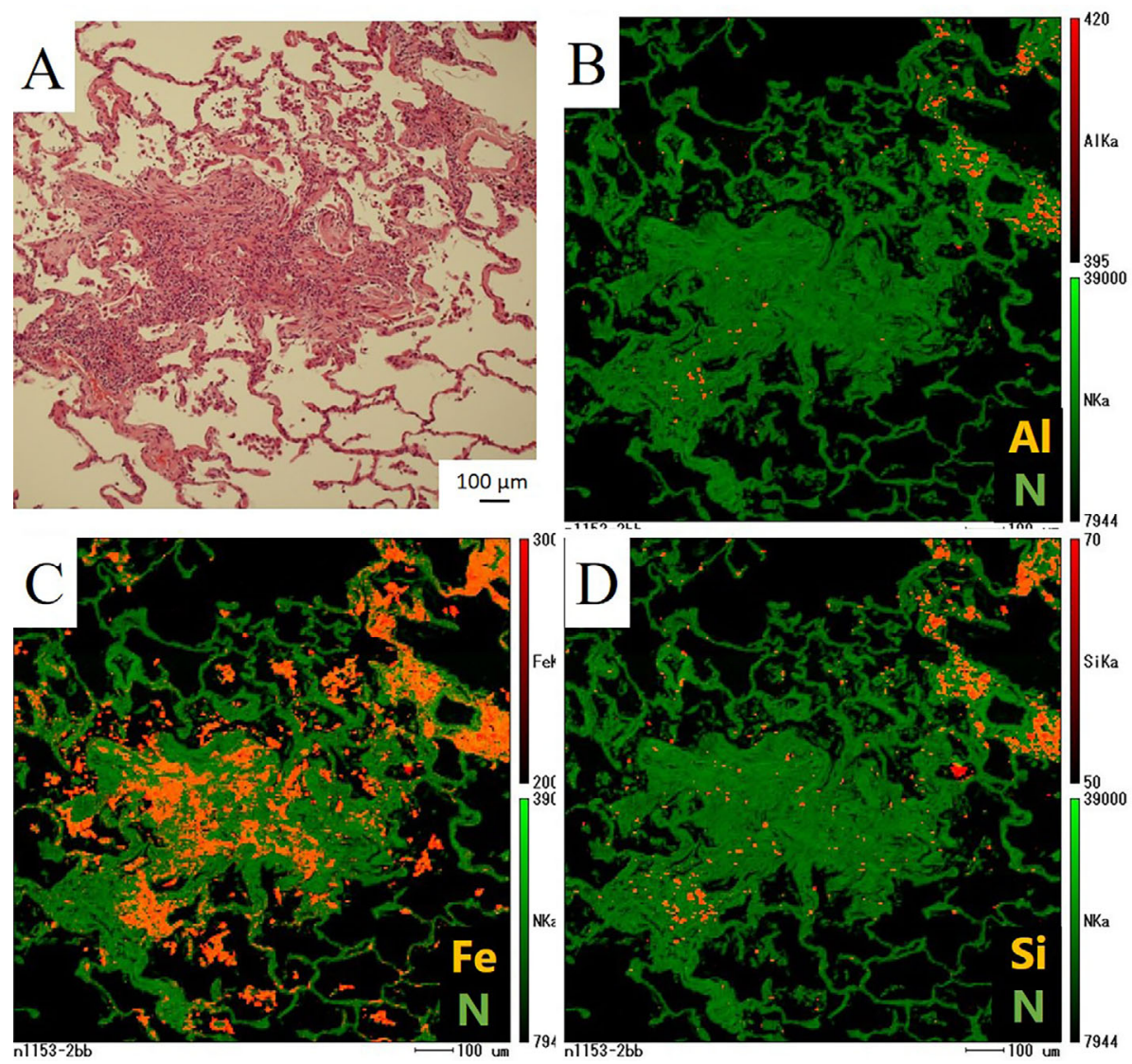

Figure 7. An electron probe microanalysis of the anterior segment of the right upper lobe in case 2. (A) Hematoxylin and Eosin staining. The deposition of aluminum (B), iron (C), and silicon (D) is shown in yellow.

Therefore, the dried sludge from the tsunami can reach the peripheral zone of the lung. In the Hanshin-Awaji earthquake, the damaged area presented high concentrations of airborne particles, particularly on sunny days (5). We believe that our patients were exposed to high concentrations of dust because most of their work had been indoors. It is also important to note that our patients did not use any countermeasures against dust, such as wearing a dust protective mask.

Earthquakes and associated tsunamis cause not only direct pulmonary complications such as chest trauma, drowning, or aspiration pneumonia, but also secondary complications via the inhalation of airborne particles from the sludge and rubble. However, only a few studies have reported on the occurrence of diffuse parenchymal lung disease associated with earthquakes or tsunamis. However, increased morbidity due to MPO-ANCA-related angiitis with respiratory involvement and one case of pulmonary alveolar proteinosis caused by restoration work after the 1995 Hanshin-Awaji Great Earthquake have been reported $(6,7)$. Furthermore, a mass outbreak of organic dust toxic syndrome after the 2004 Niigata-Chuetsu Earthquake in Japan has been reported. However, cases of OP after earthquakes and tsunamis have not yet been previously reported.

After the Great East Japan Earthquake, one case of fatal acute interstitial pneumonia in a worker that made chips from wooden debris generated after the incident together with pulmonary alveolar proteinosis due to the inhalation of dried sludge from the tsunami was reported $(8,9)$. The EPMA findings of this case were similar to those of our cases; however, the histopathology results and the clinical course of OP differed from our cases. Although these cases indicated that several individuals had inhaled the dried sludge, only some individuals developed diffuse parenchymal lung disease. Therefore, we believe that patient factors are important in such disease development; however, we did not assess these factors in this report.

One case of acute eosinophilic pneumonia in a firefighter exposed to the dust cloud produced after the collapse of the World Trade Center has also been reported (10). The firefighter inhaled high concentrations of dust during the rescue effort without any airway protective device. An elemental analysis indicated the presence of $\mathrm{Mg}, \mathrm{Si}$, and $\mathrm{Fe}$. The analysis of air samples indicated the presence of particles smaller than 2.5 micrometers. He rapidly recovered after treatment with corticosteroids. The onset and clinical course of the disease in this case were similar to those observed in our cases, but the diagnosis was different. The authors made a diagnosis based on the presence of eosinophils in the BAL fluid, but did not confirm the diagnosis via histopathological examinations.

One case of OP caused by the inhalation of volcanic ash 

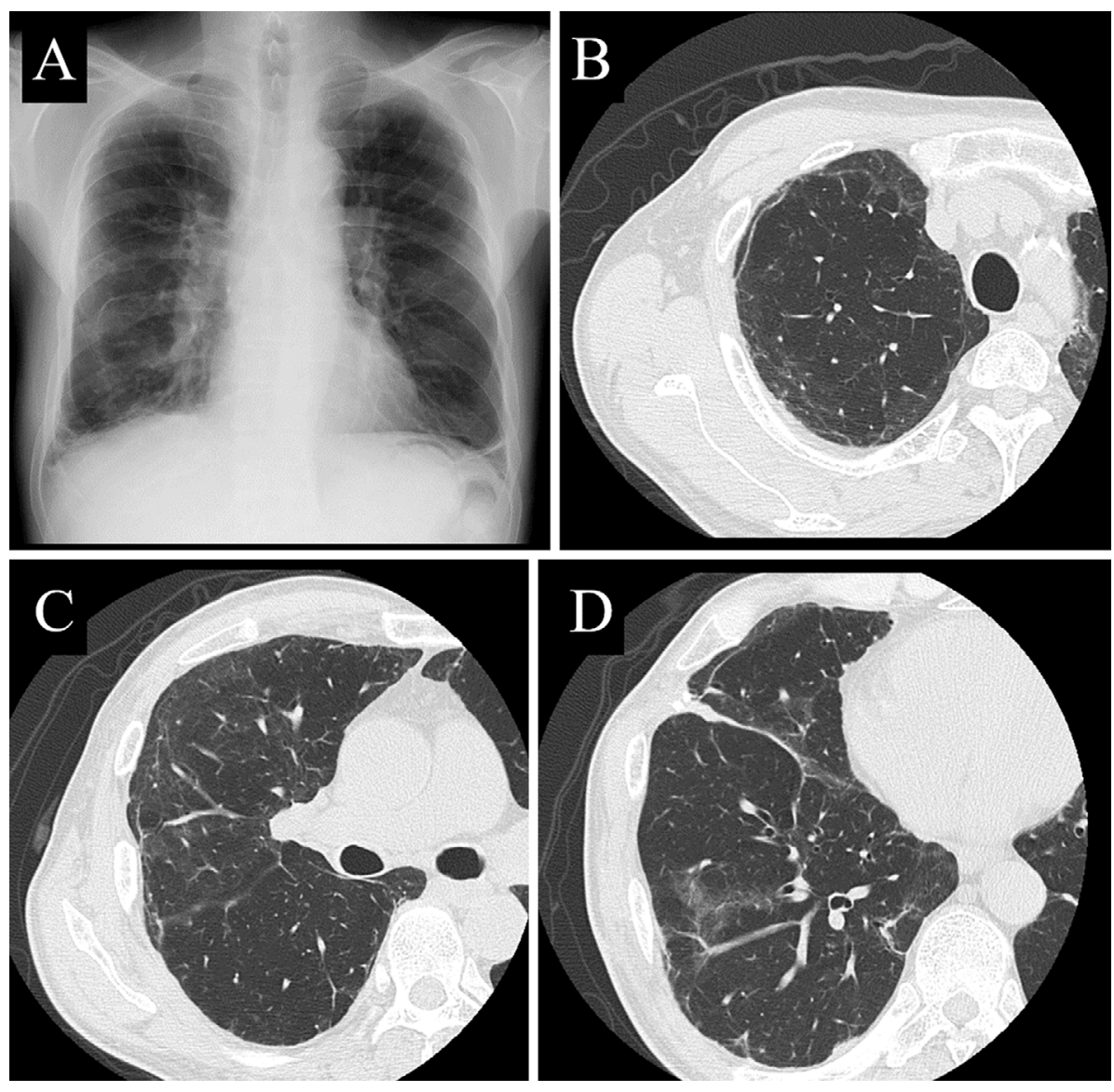

Figure 8. Chest radiograph (A) and high-resolution chest CT scan (lung windowing) of the right lobe (B-D) after treatment in case 1.

has also been reported (11). The main components of volcanic ash are oxidized $\mathrm{Fe}, \mathrm{Si}$, and $\mathrm{Al}$, which were also present in the tsunami sludge. This case has several similarities with our cases. First, this case developed after the inhalation of high concentrations of dust, which contained large amounts of $\mathrm{Fe}, \mathrm{Si}$, and Al. Second, a histopathology examination indicated the infiltration of inflammatory cells in the peribronchioles and the presence of fibrous plugs in the alveolar ducts. Third, an elemental analysis demonstrated that the inhaled dust reached the peripheral zone of the lung. Therefore, this report supports our hypothesis that the inhalation of high amounts of dust can cause OP.

Both our cases and the above three studies that performed an elemental analysis demonstrated the deposition of inorganic substances such as $\mathrm{Fe}, \mathrm{Si}$, and $\mathrm{Al}$, which are the main components of the earth crust and are known to cause pneumoconiosis but not OP. Although silica can cause acute silico-proteinosis a few months after inhalation, the histopathological findings and clinical course of this disease indicate that this disease does not respond to corticosteroids, and this result is different from that observed in our cases (12). Therefore, we could not conclude that these substances had caused OP. However, it is known that patients with silicosis suffer not only from respiratory complications, but also from autoimmune disorders such as rheumatoid arthritis $(13,14)$, systemic lupus erythematosus $(15,16)$, sys- temic sclerosis $(17,18)$, and anti-neutrophilic cytoplasmic antibody (ANCA)-associated vasculitis (19-22), and silica is known to elicit an inflammatory response characterized by the release of cytokines, such as IL- $1 \beta$ and TNF $\alpha(23,24)$. Therefore, we believe that the inhalation of high concentrations of dust that contains small-sized particles from the earth crust could thus have been the cause of OP in our cases.

Dried sludge from tsunamis contains inorganic and organic compounds. The development of OP associated with the inhalation of organic compounds has only rarely been reported. In 1992, a case of textile printing dye-related OP was described in 22 textile airbrush workers (25). It has been suggested that this complication had been caused by the spraying of a breathable aerosol containing a particular printing dye. A case of Penicillium mold dust-related OP has also been described (26). Although our patients did not use any printing dye and no fungi were detected in their lungs, the development of OP by organic compounds in our cases is also possible.

In conclusion, we herein reported two cases of OP caused by the inhalation of dried sludge from the tsunami after a large-scale earthquake. These cases emphasize the urgent need to protect individuals who engage in restoration work from dust inhalation in the aftermath of a tsunami or other similar natural disaster. 

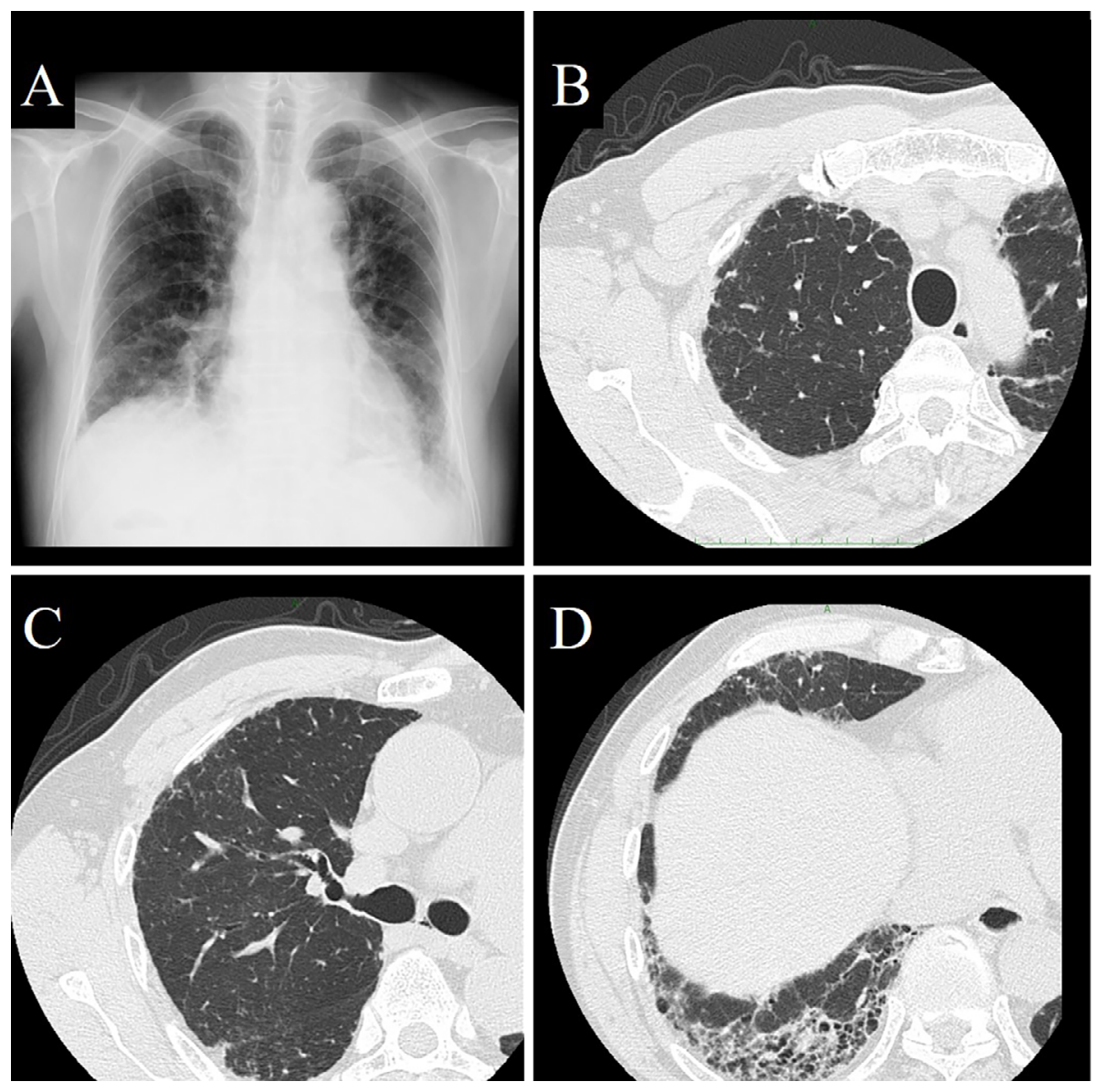

Figure 9. Chest radiograph (A) and high-resolution chest CT scan (lung windowing) of the right lobe (B-D) after treatment in case 2.

The authors state that they have no Conflict of Interest (COI).

\section{Acknowledgement}

The authors are grateful to Hiroshi Moriyama, from Niigata University, for the electron probe microanalysis of the specimens and helpful comments.

\section{References}

1. Epler GR. Bronchiolitis obliterans organizing pneumonia. Arch Intern Med 161: 158-164, 2001.

2. Yamanda S, Hanagama M, Kobayashi S, et al. The impact of the 2011 Great East Japan Earthquake on hospitalisation for respiratory disease in a rapidly aging society: a retrospective descriptive and cross-sectional study at the disaster base hospital in Ishinomaki. BMJ Open 3: e000865, 2013.

3. Geiser M, Kreyling WG. Deposition and biokinetics of inhaled nanoparticles. Part Fibre Toxicol 7: 2, 2010.

4. Blott SJ, Pye K. GRADISTAT: a grain size distribution and statistics package for the analysis of unconsolidated sediments. Earth Surf Proc Land 26: 1237-1248, 2001.

5. Yamamoto R, Nagai N, Koizumi N, Ninomiya R. Dust concentration around the sites of demolition work after the Great HanshinAwaji Earthquake. Environ Health Prev Med 3: 207-214, 1999.

6. Yashiro M, Muso E, Itoh T, Oyama A, Ono T, Sasayama S. Significantly high incidence and high morbidity of acute renal failure with respiratory tract involvement of p-ANCA-related angitis revealed in Kobe city and the environs after the Kobe earthquake in 1995. Clin Nephrol 51: 190-191, 1999.

7. Tsuchiya T, Nishimura Y, Kotani Y, et al. Secondary pulmonary al- veolar proteinosis in a patient engaged in building demolition work. Nihon Kokyuuki Gakkai Zasshi (The Journal of the Japanese Respiratory Society) 37: 219-223, 1999 (in Japanese, Abstract in English).

8. Ohkouchi S, Ebina M, Kamei K, et al. Fatal acute interstitial pneumonia in a worker making chips from wooden debris generated by the Great East Japan earthquake and tsunami. Respir Investig 50: 129-134, 2012.

9. Hisata S, Moriyama H, Tazawa R, Ohkouchi S, Ichinose M, Ebina M. Development of pulmonary alveolar proteinosis following exposure to dust after the Great East Japan Earthquake. Respir Investig 51: 212-216, 2013.

10. Rom WN, Weiden M, Garcia R, et al. Acute eosinophilic pneumonia in a New York City firefighter exposed to World Trade Center dust. Am J Respir Crit Care Med 166: 797-800, 2002.

11. Shojima J, Ikushima $S$, Ando $T$, et al. A case of volcanic ash lung: report of a case. Nihon Kokyuuki Gakkai Zasshi (The Journal of the Japanese Respiratory Society) 44: 192-196, 2006 (in Japanese, Abstract in English).

12. Buechner HA, Ansari A. Acute silico-proteinosis. A new pathologic variant of acute silicosis in sandblasters, characterized by histologic features resembling alveolar proteinosis. Dis Chest $\mathbf{5 5}$ : 274-278, 1969.

13. Stolt P, Källberg H, Lundberg I, Sjögren B, Klareskog L, Alfredsson L. Silica exposure is associated with increased risk of developing rheumatoid arthritis: results from the Swedish EIRA study. Ann Rheum Dis 64: 582-586, 2005.

14. Caplan A. Rheumatoid disease and pneumoconiosis (Caplan's syndrome). Proc R Soc Med 52: 1111-1113, 1959.

15. Yamazaki S, Yoshiike F, Hirai K, et al. Silica-associated systemic 
lupus erythematosus in an elderly man. Intern Med 46: 1867-1871, 2007.

16. Costallat LTL, De Capitani EM, Zambon L. Pulmonary silicosis and systemic lupus erythematosus in men: a report of two cases. Joint Bone Spine 69: 68-71, 2002.

17. Haustein UF, Anderegg U. Silica induced scleroderma: clinical and experimental aspects. J Rheumatol 25: 1917-1926, 1998.

18. Ben Abdelghani K, Fazaa A, Souabni L, Zakraoui L. Association of pulmonary silicosis and systemic sclerosis. BMJ Case Rep 2015: bcr2013202509, 2015.

19. Gregorini G, Tira P, Frizza J, et al. ANCA-associated diseases and silica exposure. Clin Rev Allergy Immunol 15: 21-40, 1997.

20. Tervaert JW, Stegeman CA, Kallenberg CG. Silicon exposure and vasculitis. Curr Opin Rheumatol 10: 12-17, 1998

21. Saeki T, Fujita N, Kourakata H, Yamazaki H, Miyamura S. Two cases of hypertrophic pachymeningitis associated with myeloperoxidase antineutrophil cytoplasmic autoantibody (MPO-ANCA)positive pulmonary silicosis in tunnel workers. Clin Rheumatol 23: 76-80, 2004.

22. Gómez-Puerta JA, Gedmintas L, Costenbader KH. The association between silica exposure and development of ANCA-associated vasculitis: systematic review and meta-analysis. Autoimmun Rev 12: 1129-1135, 2013.

23. Lee S, Matsuzaki H, Kumagai-Takei N, et al. Silica exposure and altered regulation of autoimmunity. Environ Health Prev Med 19: 322-329, 2014

24. Hornung V, Bauernfeind F, Halle A, et al. Silica crystals and aluminum salts activate the NALP3 inflammasome through phagosomal destabilization. Nat Immunol 9: 847-856, 2008.

25. Camus P, Nemery B. A novel cause for bronchiolitis obliterans organizing pneumonia: exposure to paint aerosols in textile workshops. Eur Respir J 11: 259-262, 1998.

26. Bates C, Read RC, Morice AH. A malicious mould. Lancet 349 : 1598, 1997.

The Internal Medicine is an Open Access article distributed under the Creative Commons Attribution-NonCommercial-NoDerivatives 4.0 International License. To view the details of this license, please visit (https://creativecommons.org/licenses/ by-nc-nd/4.0/).

(C) 2016 The Japanese Society of Internal Medicine http://www.naika.or.jp/imonline/index.html 